\title{
High-precision calculation of the 4-loop contribution to the electron $g-2$ in QED
}

\author{
Stefano Laporta ${ }^{* \dagger}$ \\ Dipartimento di Fisica e Astronomia, Università di Padova, Via Marzolo 8, I-35131 Padova, \\ Italy \\ Istituto Nazionale Fisica Nucleare, Sezione di Padova, Via Marzolo 8, I-35131 Padova, Italy \\ E-mail: stefano.laporta@pd.infn.it
}

\begin{abstract}
In this paper I briefly describe the results of the numerical evaluation of the mass-independent 4-loop contribution to the electron $g$-2 in QED with 1100 digits of precision. In particular I also show the semi-analytical fit to the numerical value, which contains harmonic polylogarithms of $e^{\frac{i \pi}{3}}, e^{\frac{2 i \pi}{3}}$ and $e^{\frac{i \pi}{2}}$, one-dimensional integrals of products of complete elliptic integrals and six finite parts of master integrals, evaluated up to 4800 digits. I give also some information about the methods and the program used.
\end{abstract}

13th International Symposium on Radiative Corrections 24-29 September, 2017

St. Gilgen, Austria

\footnotetext{
* Speaker.

${ }^{\dagger}$ The author thanks DESY for financially supporting his participation in the symposium.
} 


\section{Electron $g-2$}

An electron of mass $m$ and spin $s$ has a magnetic moment $\mu$

$$
\mu=g \frac{e \hbar}{2 m c} s
$$

where $g$ is the gyromagnetic ratio. According to the Dirac's theory[1], the electron has $g=2$. The interaction with photons shifts slightly $g$; we define the anomaly $a_{e}=(g-2) / 2$.

\section{Experimental measurements}

The current measurements of $a_{e}$ are based on the Penning trap method, developed at the University of Washington. The anomaly is expressed as the ratio of two frequencies which can measured to a very high precision. For the development of this technique, the Noble prize in Physics 1989 was awarded to H. Dehmelt. Their final results were [2]:

$$
\begin{aligned}
& a_{e^{-}}^{\text {exp }}=1159652188.4(4.3) \times 10^{-12} \\
& a_{e^{+}}^{\text {exp }}=1159652187.9(4.3) \times 10^{-12} \quad(4.3 \mathrm{ppb}),
\end{aligned}
$$

The most recent value obtained with the same technique by the Harvard group is [3, 4]:

$$
a_{e^{-}}^{e x p}=1159652180.73(.28) \times 10^{-12} \quad(0.24 \mathrm{ppb}) .
$$

\section{Theoretical expression}

In the standard model

$$
a_{e}^{S M}=a_{e}^{Q E D}+a_{e}^{\text {weak }}+a_{e}^{\text {hadr }} .
$$

The QED contribution can be split up in mass-independent and mass-dependent parts:

$$
a_{e}^{Q E D}=A_{1}+A_{2}\left(\frac{m_{e}}{m_{\mu}}\right)+A_{2}\left(\frac{m_{e}}{m_{\tau}}\right)+A_{3}\left(\frac{m_{e}}{m_{\mu}}, \frac{m_{e}}{m_{\tau}}\right) .
$$

The functions $A^{(i)}$ can be expanded in power series

$$
A_{i}=A_{i}^{(2)}\left(\frac{\alpha}{\pi}\right)+A_{i}^{(4)}\left(\frac{\alpha}{\pi}\right)^{2}+A_{i}^{(6)}\left(\frac{\alpha}{\pi}\right)^{3}+A_{i}^{(8)}\left(\frac{\alpha}{\pi}\right)^{4}+A_{i}^{(10)}\left(\frac{\alpha}{\pi}\right)^{5}+\ldots
$$

The mass-independent coefficients at 1, 2 and 3 loop are known in analytical form $[5,6,7]$.

$$
\begin{aligned}
A_{1}^{(2)}= & \frac{1}{2} \\
A_{1}^{(4)}= & \frac{197}{144}+\frac{1}{12} \pi^{2}-\frac{1}{2} \pi^{2} \ln 2+\frac{3}{4} \zeta(3)=-0.328478965579 \ldots \\
A_{1}^{(6)}= & \frac{83}{72} \pi^{2} \zeta(3)-\frac{215}{24} \zeta(5)+\frac{100}{3}\left[\left(a_{4}+\frac{1}{24} \ln ^{4} 2\right)-\frac{1}{24} \pi^{2} \ln ^{2} 2\right]-\frac{239}{2160} \pi^{4}+ \\
& \frac{139}{18} \zeta(3)-\frac{298}{9} \pi^{2} \ln 2+\frac{17101}{810} \pi^{2}+\frac{28259}{5184}=1.181241456 \ldots
\end{aligned}
$$




\begin{tabular}{|c|c|c|c|}
\hline$A_{1}^{(4)}$ & $A_{1}^{(6)}$ & $A_{1}^{(8)}$ & $A_{1}^{(10)}$ \\
\hline$-2.973 \ldots[26]$ & $1.49(20)[27]$ & $\begin{array}{ll}-1.434(138) & {[20]}\end{array}$ & $7.795(336)[25]$ \\
\hline \multirow[t]{6}{*}{$-0.328478965579 \ldots[6]$} & $1.195(26)[28]$ & $-1.5098(384)[31]$ & $6.599(223)[25]$ \\
\hline & $1.17611(42)$ [29] & $-1.7283(35) \quad[21]$ & \\
\hline & $1.181259(40) \quad[30]$ & $-1.9144(35) \quad[22]$ & \\
\hline & $1.181241456587 \ldots$ [7] & $-1.9106(20) \quad[24]$ & \\
\hline & & $-1.91298(84)$ [25] & \\
\hline & & $-1.912245764926 \ldots$ [8] & \\
\hline
\end{tabular}

Table 1: Numerical results of various evaluations of $A_{1}^{(4)}, A_{1}^{(6)}, A_{1}^{(8)}$ and $A_{1}^{(10)}$.

\begin{tabular}{lc}
\hline contribution & value in units of $10^{-12}$ \\
\hline$A_{1}^{(2)}(\alpha / \pi)$ & $1161409733.631(720)$ \\
$A_{1}^{(4)}(\alpha / \pi)^{2}$ & $-1772305.065(3)$ \\
$A_{1}^{(6)}(\alpha / \pi)^{3}$ & 14804.203 \\
$A_{1}^{(8)}(\alpha / \pi)^{4}$ & -55.667 \\
$A_{1}^{(10)}(\alpha / \pi)^{5}$ & $0.446(15)$ \\
$A_{2}^{(4)}\left(m_{e} / m_{\mu}\right)(\alpha / \pi)^{2}$ & 2.804 \\
$A_{2}^{(6)}\left(m_{e} / m_{\mu}\right)(\alpha / \pi)^{3}$ & -0.092 \\
$A_{2}^{(8)}\left(m_{e} / m_{\mu}\right)(\alpha / \pi)^{4}$ & 0.026 \\
$A_{2}^{(10)}\left(m_{e} / m_{\mu}\right)(\alpha / \pi)^{5}$ & -.0002 \\
$A_{2}^{(4)}\left(m_{e} / m_{\tau}\right)(\alpha / \pi)^{2}$ & 0.010 \\
$A_{2}^{(6)}\left(m_{e} / m_{\tau}\right)(\alpha / \pi)^{3}$ & -0.0008 \\
$a_{e}($ hadronic v.p. $)$ & $1.866(11)$ \\
$a_{e}($ hadronic v.p.,NLO) & $-0.223(1)$ \\
$a_{e}($ hadronic v.p.,NNLO $)$ & $0.028(1)$ \\
$a_{e}($ hadronic l-l $)$ & $0.035(10)$ \\
$a_{e}($ weak $)$ & $0.0297(5)$ \\
\hline
\end{tabular}

Table 2: Contributions to $a_{e}$.

where $\zeta(n)=\sum_{i=0}^{\infty} i^{-n}, a_{n}=\sum_{i=0}^{\infty} 2^{-i} i^{-n}$. In table 1 we list some older theoretical evaluations of the two, three and four loop coefficients.

I have evaluated the 4-loop contribution $A_{1}^{(8)}$ up to 1100 digits of precision [8], finalizing a twenty-year effort $[9,10,11,12,13,14,15]$ begun after the completion of the calculation of $A_{1}^{(6)}[7]$. The first digits of the result are

$$
A_{a}^{(4)}=-1.91224576492644557415264716743983005 \ldots
$$

The full-precision result is shown in table 3. The result (11) is in excellent agreement $(0.9 \sigma)$ with the numerical value

$$
A_{1}^{(8)}(\text { Ref. [25] })=-1.91298(84),
$$

latest result of a really impressive pluridecennial effort $[16,17,18,19,20,21,22,23,24,25]$, and with the independent value

$$
A_{1}^{(8)}(\text { Ref. [32] })=-1.87(12) \text {. }
$$




\begin{abstract}
$-1.91224576492644557415264716743983005406087339065872534517132984800603844398065170614$ 27608927000036315837558415331473270056378514912854539190280432705027382230434557895704 55627293099412966997602777822115784720339064151908166527097970867438115012155147972274 32216427343192797595860740500578373849607018743283140248380251922494607422985589304635 06140492252663431094424000235635688128062064549401322497759430042928883676174889923691 51808780869897052635785337537769641170245361960134975744943612684861751626068323871867 47303831505962741878015305514879400536977798369464278684326918431175889581159743566950 43304834907361342658649953116387811743475385423488364085584441882237217456706871041823 30743051744305573945961171550858961148995261266061246994073118403927472340023464969531 73548258481799822409737371077365740464513521123091242528111137215302154453721014811121 15984897088422327987972048420144512282845151658523656178659459260099173303172130286546 72123453405003491047007289244872006160442613254490690004319151982300474881814943110384 $953782994062967586787538524978194698979313216219797575067670114290489796208505078 \ldots$.
\end{abstract}

Table 3: First 1100 digits of $A_{1}^{(8)}$.

There are 12672 diagrams which contribute at 5-loop level. They have been computed through a gigantic effort [25]; the most recent value is

$$
A_{1}^{(10)}(\text { Ref. [25]) }=6.599(223) \text {. }
$$

We also note the MonteCarlo approach of Ref. [36] which might in future provide an independent value. Concerning the mass-dependent part $A_{2}(r), A_{2}^{(4)}(r)$ is known in analytical form [37], as well as $A_{2}^{(6)}(r)[38,39,40,41,42]$; the first terms of the expansion for small $r$ of the 4-loop coefficient $A_{2}^{(8)}(r)$ are known analytically $[43,44] . A_{2}^{(10)}\left(m_{e} / m_{\mu}\right)$ and $A_{2}^{(10)}\left(m_{e} / m_{\tau}\right)$ have been calculated numerically [25]; the first terms of the expansion for small mass ratios of $A_{3}^{(6)}\left(m_{e} / m_{\mu}, m_{e} / m_{\tau}\right)$ and $A_{3}^{(8)}\left(m_{e} / m_{\mu}, m_{e} / m_{\tau}\right)$ are known analytically [44]. The values of the hadronic and weak contributions $[45,46,47,48]$ are shown in table 2 , where the most precise determination of the fine structure constant $[49,50]$

$$
\alpha^{-1}=137.035998996(85) \quad(0.62 \mathrm{ppb})
$$

is used. Inserting all the contributions (see Eq.s(8-14) and table 2) into Eq.s(5-7) one finds

$$
a_{e}^{\text {th }}=1159652182.031(15)(15)(720) \times 10^{-12},
$$

where the first error comes from $A_{1}^{(10)}$, the second one from the hadronic and electroweak corrections, the last one from $\alpha$. Conversely, assuming the validity of the theory and using the experimental measurement (4), one finds

$$
\alpha^{-1}\left(a_{e}\right)=137.0359991500(18)(18)(330) \quad(0.25 \mathrm{ppb})
$$

where the errors come respectively from $A_{1}^{(10)}$, hadronic and electroweak corrections, and the experimental measurement. This determination of $\alpha$ is more precise than the experimental one and is that used in the periodic least-squares adjustments of fundamental constants[48].

\title{
Gauge-invariant sets
}

The contributions to $g$ - 2 of single Feynman diagrams may be I.R. or U.V. divergent, and depends on the gauge chosen. Therefore, it is convenient to regroup the diagrams in gauge invariant 
sets, whose contributions are finite. The number of diagrams at 2, 3 and 4 loops are respectively 7 , 72 and 891; they can be arranged in, respectively 3, 9 (see Ref. [51]) and 25 gauge invariant sets. Typical elements of the sets at four loops are shown in Fig.1; the values of the contributions of each gauge set are shown in Ref. [8] with 40 digits of precision.

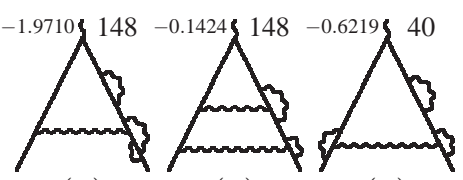

(1)
(2)

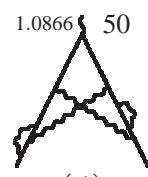

(4)

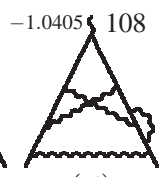

(5)

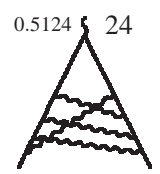

(6)

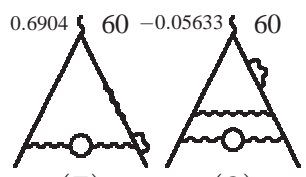

(7)

(8)

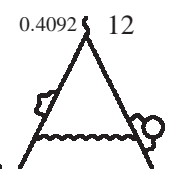

(9)

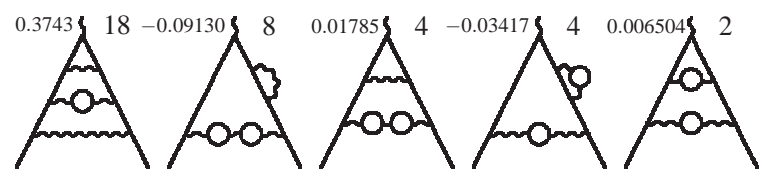

(10)
(11)
(13)

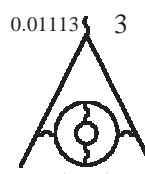

(19)

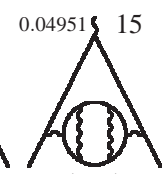

(20)
(12)

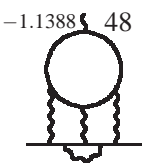

(21)

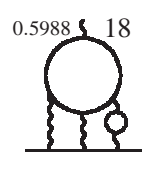

(22)
(14)

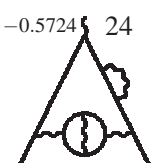

(15)

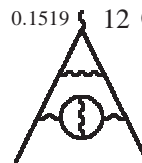

(16)

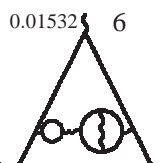

(17)

Figure 1: Typical representative diagrams of gauge-invariant sets. For each set only one diagrams is shown. Top left: contribution of the set to $g$-2; top right: number of diagrams of the set

\section{The numerical fit}

By using the integration-by-parts identities[52, 53, 9] the contributions of all 4-loop vertex diagrams can be reduced to a linear combination of 334 master integrals belonging to 220 topologies. Due to the expected analytical complexity of the result, a completely analytical calculation of all the master integrals (for example similar to that at three-loop) has seemed out of reach. So I have used a different approach: compute very high-precision numerical values of a sufficient number of terms of the expansions of the master integrals in Laurent series of $\varepsilon=(4-D) / 2$ dimensions; make the right analytical ansatz on the analytical form; fit the rational coefficients of the ansatz by using the PSLQ algorithm [54, 55].

As a result, one finds that the analytical expressions of the coefficients of the $\varepsilon$-expansions of the master integrals contain values of harmonic polylogarithms [57] with argument $1, \frac{1}{2}, e^{\frac{i \pi}{3}}, e^{\frac{2 i \pi}{3}}$ and $e^{\frac{i \pi}{2}}$, a family of one-dimensional integrals of products of elliptic integrals, and the finite terms of the $\varepsilon$-expansions of six master integrals. Work is still in progress to fit analytically these six unknown elliptical constants. The result of the analytical fit for $A_{1}^{(8)}$ can be written as follows:

$$
A_{1}^{(8)}=T+\sqrt{3} V_{a}+V_{b}+W_{b}+\sqrt{3} E_{a}+E_{b}+U
$$




$$
\begin{aligned}
& T=\frac{1243127611}{130636800}+\frac{30180451}{25920} \zeta(2)-\frac{255842141}{2721600} \zeta(3)-\frac{8873}{3} \zeta(2) \ln 2+\frac{6768227}{2160} \zeta(4)+\frac{19063}{360} \zeta(2) \ln ^{2} 2 \\
& +\frac{12097}{90}\left(a_{4}+\frac{1}{24} \ln ^{4} 2\right)-\frac{2862857}{6480} \zeta(5)-\frac{12720907}{64800} \zeta(3) \zeta(2)-\frac{221581}{2160} \zeta(4) \ln 2 \\
& +\frac{9656}{27}\left(a_{5}+\frac{1}{12} \zeta(2) \ln ^{3} 2-\frac{1}{120} \ln ^{5} 2\right)+\frac{191490607}{46656} \zeta(6)+\frac{10358551}{43200} \zeta^{2}(3)-\frac{40136}{27} a_{6}+\frac{26404}{27} b_{6} \\
& -\frac{700706}{675} a_{4} \zeta(2)-\frac{26404}{27} a_{5} \ln 2+\frac{26404}{27} \zeta(5) \ln 2-\frac{63749}{50} \zeta(3) \zeta(2) \ln 2-\frac{40723}{135} \zeta(4) \ln ^{2} 2+\frac{13202}{81} \zeta(3) \ln ^{3} 2 \\
& -\frac{253201}{2700} \zeta(2) \ln ^{4} 2+\frac{7657}{1620} \ln ^{6} 2+\frac{2895304273}{435456} \zeta(7)+\frac{670276309}{193536} \zeta(4) \zeta(3)+\frac{85933}{63} a_{4} \zeta(3)-\frac{142793}{18} a_{5} \zeta(2) \\
& +\frac{7121162687}{967680} \zeta(5) \zeta(2)-\frac{195848}{21} a_{7}+\frac{195848}{63} b_{7}-\frac{116506}{189} d_{7}-\frac{4136495}{384} \zeta(6) \ln 2-\frac{1053568}{189} a_{6} \ln 2 \\
& +\frac{233012}{189} b_{6} \ln 2+\frac{407771}{432} \zeta^{2}(3) \ln 2-\frac{8937}{2} a_{4} \zeta(2) \ln 2+\frac{833683}{3024} \zeta(5) \ln ^{2} 2-\frac{3995099}{6048} \zeta(3) \zeta(2) \ln ^{2} 2 \\
& -\frac{233012}{189} a_{5} \ln ^{2} 2+\frac{1705273}{1512} \zeta(4) \ln ^{3} 2+\frac{602303}{4536} \zeta(3) \ln ^{4} 2-\frac{1650461}{11340} \zeta(2) \ln ^{5} 2+\frac{52177}{15876} \ln ^{7} 2, \\
& V_{a}=-\frac{14101}{480} \mathrm{Cl}_{4}\left(\frac{\pi}{3}\right)-\frac{169703}{1440} \zeta(2) \mathrm{Cl}_{2}\left(\frac{\pi}{3}\right)+\frac{494}{27} \operatorname{Im} H_{0,0,0,1,-1,-1}\left(e^{i \frac{\pi}{3}}\right)+\frac{494}{27} \operatorname{Im} H_{0,0,0,1,-1,1}\left(e^{i \frac{2 \pi}{3}}\right) \\
& +\frac{494}{27} \operatorname{Im} H_{0,0,0,1,1,-1}\left(e^{i \frac{2 \pi}{3}}\right)+19 \operatorname{Im} H_{0,0,1,0,1,1}\left(e^{i \frac{2 \pi}{3}}\right)+\frac{437}{12} \operatorname{Im} H_{0,0,0,1,1,1}\left(e^{i \frac{2 \pi}{3}}\right)+\frac{29812}{297} \mathrm{Cl}_{6}\left(\frac{\pi}{3}\right) \\
& +\frac{4940}{81} a_{4} \mathrm{Cl}_{2}\left(\frac{\pi}{3}\right)-\frac{520847}{69984} \zeta(5) \pi-\frac{129251}{81} \zeta(4) \mathrm{Cl}_{2}\left(\frac{\pi}{3}\right)-\frac{892}{15} \operatorname{Im} H_{0,1,1,-1}\left(e^{i \frac{2 \pi}{3}}\right) \zeta(2) \\
& -\frac{1784}{45} \operatorname{Im} H_{0,1,1,-1}\left(e^{i \frac{\pi}{3}}\right) \zeta(2)+\frac{1729}{54} \zeta(3) \operatorname{Im} H_{0,1,-1}\left(e^{i \frac{\pi}{3}}\right)+\frac{1729}{36} \zeta(3) \operatorname{Im} H_{0,1,1}\left(e^{i \frac{2 \pi}{3}}\right) \\
& +\frac{837190}{729} \mathrm{Cl}_{4}\left(\frac{\pi}{3}\right) \zeta(2)+\frac{25937}{4860} \zeta(3) \zeta(2) \pi-\frac{223}{243} \zeta(4) \pi \ln 2+\frac{892}{9} \operatorname{Im} H_{0,1,-1}\left(e^{i \frac{\pi}{3}}\right) \zeta(2) \ln 2 \\
& +\frac{446}{3} \operatorname{Im} H_{0,1,1}\left(e^{i \frac{2 \pi}{3}}\right) \zeta(2) \ln 2-\frac{7925}{81} \mathrm{Cl}_{2}\left(\frac{\pi}{3}\right) \zeta(2) \ln ^{2} 2+\frac{1235}{486} \mathrm{Cl}_{2}\left(\frac{\pi}{3}\right) \ln ^{4} 2, \\
& V_{b}=\frac{13487}{60} \operatorname{Re} H_{0,0,0,1,0,1}\left(e^{i \frac{\pi}{3}}\right)+\frac{13487}{60} \mathrm{Cl}_{4}\left(\frac{\pi}{3}\right) \mathrm{Cl}_{2}\left(\frac{\pi}{3}\right)+\frac{136781}{360} \mathrm{Cl}_{2}^{2}\left(\frac{\pi}{3}\right) \zeta(2)+\frac{651}{4} \operatorname{Re}_{0,0,0,1,0,1,-1}\left(e^{i \frac{\pi}{3}}\right) \\
& +651 \operatorname{Re} H_{0,0,0,0,1,1,-1}\left(e^{i \frac{\pi}{3}}\right)-\frac{17577}{32} \operatorname{Re} H_{0,0,1,0,0,1,1}\left(e^{i \frac{2 \pi}{3}}\right)-\frac{87885}{64} \operatorname{Re} H_{0,0,0,1,0,1,1}\left(e^{i \frac{2 \pi}{3}}\right) \\
& -\frac{17577}{8} \operatorname{Re}_{0,0,0,0,1,1,1}\left(e^{i \frac{2 \pi}{3}}\right)+\frac{651}{4} \mathrm{Cl}_{4}\left(\frac{\pi}{3}\right) \operatorname{Im} H_{0,1,-1}\left(e^{i \frac{\pi}{3}}\right)+\frac{1953}{8} \mathrm{Cl}_{4}\left(\frac{\pi}{3}\right) \operatorname{Im} H_{0,1,1}\left(e^{i \frac{2 \pi}{3}}\right) \\
& +\frac{31465}{176} \mathrm{Cl}_{6}\left(\frac{\pi}{3}\right) \pi+\frac{211}{4} \operatorname{Re} H_{0,1,0,1,-1}\left(e^{i \frac{\pi}{3}}\right) \zeta(2)+\frac{211}{2} \operatorname{Re} H_{0,0,1,1,-1}\left(e^{i \frac{\pi}{3}}\right) \zeta(2) \\
& +\frac{1899}{16} \operatorname{Re} H_{0,1,0,1,1}\left(e^{i \frac{2 \pi}{3}}\right) \zeta(2)+\frac{1899}{8} \operatorname{Re} H_{0,0,1,1,1}\left(e^{i \frac{2 \pi}{3}}\right) \zeta(2)+\frac{211}{4} \operatorname{Im} H_{0,1,-1}\left(e^{i \frac{\pi}{3}}\right) \mathrm{Cl}_{2}\left(\frac{\pi}{3}\right) \zeta(2) \\
& +\frac{633}{8} \operatorname{Im} H_{0,1,1}\left(e^{i \frac{2 \pi}{3}}\right) \mathrm{Cl}_{2}\left(\frac{\pi}{3}\right) \zeta(2), \\
& W_{b}=\zeta(2)\left(-\frac{28276}{25} \mathrm{Cl}_{2}\left(\frac{\pi}{2}\right)^{2}+104\left(4 \mathrm{Re} H_{0,1,0,1,1}(i)+4 \operatorname{Im} H_{0,1,1}(i) \mathrm{Cl}_{2}\left(\frac{\pi}{2}\right)-2 \mathrm{Cl}_{4}\left(\frac{\pi}{2}\right) \pi+\mathrm{Cl}_{2}^{2}\left(\frac{\pi}{2}\right) \ln 2\right)\right), \\
& E_{a}=\pi\left(-\frac{28458503}{691200} B_{3}+\frac{250077961}{18662400} C_{3}\right)+\frac{483913}{77760} \pi f_{2}(0,0,1)+\pi\left(\frac{4715}{1944} \ln 2 f_{2}(0,0,1)+\frac{270433}{10935} f_{2}(0,2,0)\right. \\
& \left.-\frac{188147}{4860} f_{2}(0,1,1)+\frac{188147}{12960} f_{2}(0,0,2)\right)+\pi\left(\frac{826595}{248832} \zeta(2) f_{2}(0,0,1)-\frac{5525}{432} \ln 2 f_{2}(0,0,2)\right. \\
& +\frac{5525}{162} \ln 2 f_{2}(0,1,1)-\frac{5525}{243} \ln 2 f_{2}(0,2,0)+\frac{526015}{248832} f_{2}(0,0,3)-\frac{4675}{768} f_{2}(0,1,2)+\frac{1805965}{248832} f_{2}(0,2,1) \\
& \left.-\frac{3710675}{1119744} f_{2}(0,3,0)-\frac{75145}{124416} f_{2}(1,0,2)-\frac{213635}{124416} f_{2}(1,1,1)+\frac{168455}{62208} f_{2}(1,2,0)+\frac{69245}{124416} f_{2}(2,1,0)\right) \text {, } \\
& E_{b}=\zeta(2)\left(\frac{2541575}{82944} f_{1}(0,0,2)-\frac{556445}{6912} f_{1}(0,1,1)+\frac{54515}{972} f_{1}(0,2,0)-\frac{75145}{20736} f_{1}(1,0,1)\right)-\frac{4715}{1458} \zeta(2) f_{1}(0,0,1)
\end{aligned}
$$




$$
U=-\frac{541}{300} C_{81 a}-\frac{629}{60} C_{81 b}+\frac{49}{3} C_{81 c}-\frac{327}{160} C_{83 a}+\frac{49}{36} C_{83 b}+\frac{37}{6} C_{83 c} .
$$

$C_{8 x y}$ are the $\varepsilon^{0}$ coefficients of the $\varepsilon$-expansion of six master integrals (see $f, f^{\prime}, f^{\prime \prime}, g, g^{\prime}, g^{\prime \prime}$ of Fig.2); they appear only in the gauge-invariant sets 24 and 25 . In the above expressions $b_{6}=$ $H_{0,0,0,0,1,1}\left(\frac{1}{2}\right), b_{7}=H_{0,0,0,0,0,1,1}\left(\frac{1}{2}\right), d_{7}=H_{0,0,0,0,1,-1,-1}(1), \mathrm{Cl}_{n}(\theta)=\operatorname{ImLi}_{n}\left(e^{i \theta}\right) . H_{i_{1}, i_{2}, \ldots}(x)$ are the harmonic polylogarithms. The integrals $f_{j}$ are defined as follows:

$$
\begin{aligned}
f_{m}(i, j, k) & =\int_{1}^{9} d s D_{1}(s) \operatorname{Re}\left(\sqrt{3^{m-1}} D_{m}(s)\right)\left(s-\frac{9}{5}\right) \ln ^{i}(9-s) \ln ^{j}(s-1) \ln ^{k}(s), \\
D_{m}(s) & =\frac{2}{\sqrt{(\sqrt{s}+3)(\sqrt{s}-1)^{3}}} K\left(m-1-(2 m-3) \frac{(\sqrt{s}-3)(\sqrt{s}+1)^{3}}{(\sqrt{s}+3)(\sqrt{s}-1)^{3}}\right)
\end{aligned}
$$

$K(x)$ is the complete elliptic integral of the first kind. The constants $B_{3}$ and $C_{3}$, defined in Ref. [14], admit the hypergeometric representations:

$$
\begin{aligned}
& B_{3}=\frac{4 \pi^{\frac{3}{2}}}{3}\left(\frac{\Gamma^{2}\left(\frac{7}{6}\right) \Gamma\left(\frac{1}{3}\right)}{\Gamma^{2}\left(\frac{2}{3}\right) \Gamma\left(\frac{5}{6}\right)} 4 F_{3}\left(\begin{array}{c}
\frac{1}{6} \frac{1}{3} \frac{1}{3} \frac{1}{2} \\
\frac{5}{6} \frac{5}{6} \frac{2}{3}
\end{array} ;\right) \frac{\Gamma^{2}\left(\frac{5}{6}\right) \Gamma\left(-\frac{1}{3}\right)}{\Gamma^{2}\left(\frac{1}{3}\right) \Gamma\left(\frac{1}{6}\right)} 4 F_{3}\left(\begin{array}{c}
\frac{1}{2} \frac{2}{3} \frac{2}{3} \frac{5}{6} \\
\frac{7}{6} \frac{7}{6} \frac{4}{3}
\end{array} ;\right)\right),
\end{aligned}
$$

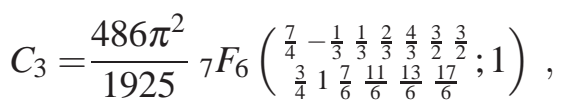

Fig.2 shows the fundamental elliptic master integrals which contain irreducible combinations of $B_{3}, C_{3}$ and $f_{m}(i, j, k)$.

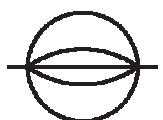

(a)

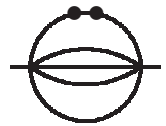

$\left(a^{\prime}\right)$

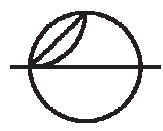

(b)

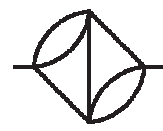

(c)

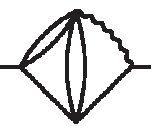

$(d)$

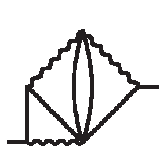

(e)

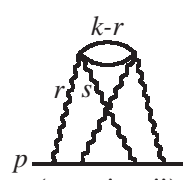

$\frac{f\left(f, f^{\prime}, f^{\prime \prime}\right)}{(1)}$

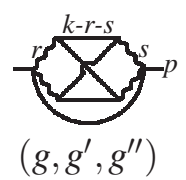

Figure 2: Minimal set of master integrals which contain all the elliptic constants. The double dot in $\left(a^{\prime}\right)$ means that denominator is raised to the power three. $\left(f, f^{\prime}, f^{\prime \prime}\right)$ and $\left(g, g^{\prime}, g^{\prime \prime}\right)$ have numerators respectively equal to $\left(1, p \cdot k,(p \cdot k)^{2}\right)$.

More in detail, the $\varepsilon$-expansions of the master integrals were first calculated with a precision of 200-300 digits, then recalculated with a precision of 4096 bit (1233 digits). A first run of PSLQ on the coefficients with a trial basis identified which coefficients are linearly independent (that is, which contain new analytical terms). For each of these "unknown constants", the simplest master integral which contains it was identified; these special master integral were recalculated with a precision of 8,16 or 32 kbits (2464, 4932 or 9864 digits). The structure the master integrals was analysed to guess the analytical form of the new elements; several runs of PSLQ with basis containing (all or selections of) these new elements were performed until the PSLQ fit was successful and the correct basis was found. 
To give an example, the PSLQ basis needed to fit $T$ of Eq. 17 contains $F_{10}-1=54$ terms ( $F_{i}$ are the Fibonacci numbers, $F_{1}=F_{2}=1$ ). Due to the presence of harmonic polylogarithms of complex argument, the general basis needed to perform the fit of $V_{a}$ and $V_{b}$ contains $F_{17}-1=1596$ terms. The practical maximum size of basis usable in PSLQ fits turned out to be $\sim 500$, so some guessing has been necessary to identify which elements are really needed in the fits (for example, only 93 elements for $V_{a}$ and $V_{b}$ ). The precision of the calculation of elements of the basis is up to 9864 digits. We note that the multi-pair parallel version [55] of the PSLQ algorithm has been essential to work out these difficult analytical fits in reasonable times.

\section{Method of calculation}

All the 104 4-loop self-mass diagrams are generated with a $C$ program; for each self-mass diagram, the corresponding vertex diagrams are generated by inserting a photon in all possible ways. For the sake of subsequent checks, one keeps also the diagrams which give no contribution because of Furry's theorem. The contribution to $g-2$ of the diagram is extracted from the unrenormalized amplitude of each vertex diagram using suitable projectors, with a FORM[56] program. The results are expressions which typically contain 100-30000 different Feynman integrals. The total number of topologically distinct master integrals is 334 . There are 82 topologies which have more than one master integral. For these topologies, products of scalar products are chosen as numerators, the choice depending on the momentum flow of the diagram. For the sake of subsequent checks, one processes separately the contributions from each one of the 104 self-mass diagrams. The master integrals are calculated separately for each self-mass diagram; therefore the total number of the master integrals actually calculated is 4607 . Because of differences in momentum routing and therefore in the numerators, topologies with more than one master integral will have a slightly different set of master integrals in different self-mass diagrams. This provides a lot of useful checks. The reduction is exact in $D$, and is performed by generating large systems of integration-by-parts identities and solving them with the algorithm [9] implemented in SYS. These large systems contain $4 \cdot 10^{6}-50 \cdot 10^{6}$ identities, with physical disk space ranging from 4GB to $100 \mathrm{~GB}$. The reduction to master integrals has been repeated with 32 and 64 bit versions of SYS. In addition, the principal self-mass diagrams were reprocessed using a different momentum flow, checking that reduction to master integrals remained the same (after converting different sets of master integrals).

For the numerical calculation of master integrals I have used combinations of the the difference equation method $[9,10]$ and the differential equation method $[59,60,61]$. An approach consists in calculating with difference equations (inserting the exponent $n$ of the first electron propagator) the integral obtained putting the photon mass $\lambda$ equal to the electron mass $m$, and integrating a differential equation in $\lambda$ from $\lambda=m$ to $\lambda=0$. A second approach consists in calculating with difference equations the diagram obtained by putting the the external momentum $p=0$, and integrating a differential equation in $p^{2}$ from $p^{2}=0$ to $p^{2}=-m^{2}$. The systems of difference and differential equations for master integrals are obtained by building systems of integration-by-parts identities and solving them by using the algorithm $[9,10]$ using rational arithmetic in $D$. The sizes of the systems of difference or differential equations to be numerically solved are in the range 1MB-3GB. Difference equations are solved using the Laplace transformation method (integral representation of solutions and differential equation of the integrand). Differential equations are solved numeri- 
cally, by using series expansion with truncated expansions in $\varepsilon=(4-D) / 2$ as coefficients. The minimum number of terms of the expansion in $\varepsilon$ is $9\left(\varepsilon^{-4} \ldots \varepsilon^{4}\right)$. There are cancellations of $\varepsilon$ terms in intermediate steps; no care is used to avoid cancellations, as the corresponding numerical zeroes provide extremely useful checks. In the worst case 37 terms of expansions are needed. The standard precision of calculations is $4 \mathrm{kbit}$ (1233 digits). About 130 digits are lost due to cancellations, so that the result has 1100 digits exact. Note that the analytical fit of some selected master integrals required a precision much higher, up to $16 \mathrm{kbit}$ (9864 digits).

The renormalization counterterms are generated with two procedures written in $C$ and FORM. Their expressions are reduced to master integrals already known in analytical form. I have chosen the Feynman gauge. I checked explicitly the (internal) gauge invariance of the contributions for arbitrary gauge of the photon line going into 1- ,2- or 3-loop vacuum polarization diagrams.

In order to perform this calculation, in 1995 I begun writing from scratch a comprehensive $C$ program, SYS [9], now 24000 lines long, containing all the parts necessary to the calculations, like a simplified fast algebraic symbolic manipulator, a numerical solver of systems of difference and differential equations, and a library of arbitrary precision mathematical routines, for integer and floating point numbers, independent of any other existing in the literature.. Another important feature is the thorough protection of large buffers and I/O with checksums. Throughout the calculation, I have discovered several subtle corruptions, due to different causes, like marginal coupling of non-ECC RAM modules (error-rate: 1 bit per week), failing RAID systems (error-rate: one corrupted 64KB block every 100GB of data ), etc.... The prompt identification and elimination of the source of errors has resulted in an highly reliable result.

\section{References}

[1] P. A. M. Dirac, Proc. Roy. Soc. Lond. A 117 (1928) 610.

[2] R. S. Van Dyck, P. B. Schwinberg and H. G. Dehmelt, Phys. Rev. Lett. 59 (1987) 26.

[3] D. Hanneke, S. Fogwell and G. Gabrielse, Phys. Rev. Lett. 100 (2008) 120801.

[4] D. Hanneke, S. F. Hoogerheide and G. Gabrielse, Phys. Rev. A 83 (2011) 052122.

[5] J. Schwinger, Phys. Rev. 73 (1948) 416.

[6] A. Petermann, Helv. Phys. Acta 30 (1957) 407; C.M. Sommerfield, Phys. Rev. 107 (1957) 328.

[7] S. Laporta and E. Remiddi, Phys. Lett. B 379 (1996) 283.

[8] S. Laporta, Phys. Lett. B 772 (2017) 232.

[9] S. Laporta, Int. J. Mod. Phys. A 15 (2000) 5087.

[10] S. Laporta, Phys. Lett. B 504 (2001) 188.

[11] S. Laporta, Phys. Lett. B 523 (2001) 95.

[12] S. Laporta, Acta Phys. Polon. B 34 (2003) 5323.

[13] S. Laporta, P. Mastrolia and E. Remiddi, Nucl. Phys. B 688 (2004) 165.

[14] S. Laporta, Int. J. Mod. Phys. A 23 (2008) 5007.

[15] S. Laporta, Subnucl. Ser. 45 (2009) 409.

[16] T. Kinoshita and W. B. Lindquist, Phys. Rev. D 27 (1983) 867. 
[17] T. Kinoshita and W. B. Lindquist, Phys. Rev. D 27 (1983) 877.

[18] T. Kinoshita and W. B. Lindquist, Phys. Rev. D 27 (1983) 886.

[19] T. Kinoshita and W. B. Lindquist, Phys. Rev. D 39 (1989) 2407.

[20] T. Kinoshita and W. B. Lindquist, Phys. Rev. D 42 (1990) 636.

[21] T. Kinoshita and M. Nio, Phys. Rev. D 73 (2006) 013003.

[22] T. Aoyama, M. Hayakawa, T. Kinoshita and M. Nio, Phys. Rev. Lett. 99 (2007) 110406.

[23] T. Aoyama, M. Hayakawa, T. Kinoshita and M. Nio, Phys. Rev. D 77, (2008) 053012.

[24] T. Aoyama, M. Hayakawa, T. Kinoshita and M. Nio, Phys. Rev. Lett. 109 (2012) 111807.

[25] T. Aoyama, M. Hayakawa, T. Kinoshita and M. Nio, Phys. Rev. D 91033006 (2015) ; Erratum: Phys. Rev. D 96 (2017) 019901.

[26] R. Karplus and N. M. Kroll, Phys. Rev. 77 (1950) 536.

[27] M. J. Levine and J. Wright, Phys. Rev. Lett. 26 (1971) 1351.

[28] P. Cvitanovic and T. Kinoshita, Phys. Rev. D 10 (1974) 4007.

[29] T. Kinoshita, in Quantum Electrodynamics, edited by T. Kinoshita (World Scientific, Singapore, 1990), pp. 218-321.

[30] T. Kinoshita, Phys. Rev. Lett. 75 (1995) 4728.

[31] V. W. Hughes and T. Kinoshita, Rev. Mod. Phys. 71 (1999) S133.

[32] P. Marquard, A. V. Smirnov, V. A. Smirnov, M. Steinhauser and D. Wellmann, arXiv:1708.07138 [hep-ph]

[33] T. Kinoshita and M. Nio, Phys. Rev. D 73 (2006) 053007

[34] T. Kinoshita, T. Aoyama, M. Hayakawa and M. Nio, Nucl. Phys. Proc. Suppl. 160 (2006) 235.

[35] M. Nio, T. Aoyama, M. Hayakawa and T. Kinoshita, Nucl. Phys. Proc. Suppl. 169 (2007) 238.

[36] S. A. Volkov, J. Exp. Theor. Phys. 122 (2016) 1008; Zh. Eksp. Teor. Fiz. 149 (2016) 1164.

[37] H. H. Elend, Phys. Lett. 20 (1966) 682.

[38] M. A. Samuel and G. w. Li, Phys. Rev. D 44 (1991) 3935 ; Erratum: Phys. Rev. D 48 (1993) 1879.

[39] G. Li, R. Mendel and M. A. Samuel, Phys. Rev. D 47 (1993) 1723.

[40] S. Laporta and E. Remiddi, Phys. Lett. B 301 (1993) 440.

[41] S. Laporta, Nuovo Cim. A 106 (1993) 675.

[42] M. Passera, Phys. Rev. D 75 (2007) 013002.

[43] A. L. Kataev, Phys. Rev. D 86 (2012) 013010.

[44] A. Kurz, T. Liu, P. Marquard and M. Steinhauser, Nucl. Phys. B 879 (2014) 1.

[45] D. Nomura and T. Teubner, Nucl. Phys. B 867 (2013) 236.

[46] A. Kurz, T. Liu, P. Marquard and M. Steinhauser, Phys. Lett. B 734 (2014) 144.

[47] J. Prades, E. de Rafael, and A Vainshtein, in Lepton Dipole Moments, edited by B. L. Roberts and W. J. Marciano (World Scientific, Singapore, 2009), 303. 
[48] P. J. Mohr, B. N. Taylor and D. B. Newell, Rev. Mod. Phys. 84 (2012) 1527.

[49] R. Bouchendira, P. Clade, S. Guellati-Khelifa, F. Nez and F. Biraben, Phys. Rev. Lett. 106 (2011) 080801 .

[50] P. J. Mohr, D. B. Newell and B. N. Taylor, Rev. Mod. Phys. 88 (2016) 035009.

[51] P. Cvitanovic, Nucl. Phys. B 127 (1977) 176.

[52] K. G. Chetyrkin and F. V. Tkachov, Nucl. Phys. B 192 (1981) 159.

[53] F. V. Tkachov, Phys. Lett. 100B (1981) 65.

[54] H. R. P. Ferguson and D. H. Bailey, RNR Technical Report RNR-91-032.

[55] D. H. Bailey and D. J. Broadhurst, Math. Comput. 70 (2001) 1719.

[56] J. A. M. Vermaseren, math-ph/0010025

[57] E. Remiddi and J. A. M. Vermaseren, Int. J. Mod. Phys. A15 (2000) 725.

[58] S. Laporta and E. Remiddi, Nucl. Phys. B 704 (2005) 349.

[59] A. V. Kotikov, Phys. Lett. B 254 (1991) 158.

[60] E. Remiddi, Nuovo Cim. A 110 (1997) 1435.

[61] T. Gehrmann and E. Remiddi, Nucl. Phys. B 580 (2000) 485. 